\title{
Role of Magnetic Resonance Imaging in Obstructive Jaundice
}

\author{
Mekala Krishna ${ }^{1}$, H B Suresh², Uthsavi N Patel ${ }^{3}$, Mahesh ${ }^{4}$ \\ ${ }^{1}$ Junior Resident, Department of Radiodiagnosis, ${ }^{2} \mathrm{Head}$ of Department (HOD), Department of Radiodiagnosis, ${ }^{3}$ Senior \\ Resident, Department of Radiodiagnosis, ${ }^{4}$ Senior Resident, Department of Radiodiagnosis, Father Muller Medical College, \\ Mangalore, India
}

Corresponding author: Dr. Uthsavi N Patel, Mullers Nest, Flat No - 605, Father Mullers Road, Souterpet, Kankanady, Mangalore, Karnataka 575002, India

DOI: http://dx.doi.org/10.21276/ijcmsr.2019.4.3.46

How to cite this article: Mekala Krishna, H B Suresh, Uthsavi N Patel, Mahesh. Role of magnetic resonance imaging in obstructive jaundice. International Journal of Contemporary Medicine Surgery and Radiology. 2019;4(3):C213-C216.

\section{A B S T R A C T}

Introduction: Jaundice is a common problem in both medical and surgical practice. The management of obstructive jaundice poses diagnostic and therapeutic challenges. MRCP is an application of MR imaging that can provide both high quality cross sectional images of ductal structures and projectional (coronal) images of the biliary tree and pancreatic duct. The purpose of this study was to determine the effectiveness of MRCP in the evaluation of obstructive jaundice.

Material and methods: This was a prospective study, conducted from December 2018 to May 2019 in the department of Radiodiagnosis at Father muller medical college, Mangalore. Thirty patients in the age group of 21-85 years suspected of obstructive jaundice on the basis of clinical signs, laboratory workup were prospectively included. MRCP was done, followed up to surgery and the histopahological diagnoses and the findings were compared

Results: Of the 30 patients, 12 had choledocholithiasis, 3 had benign strictures, 1 had primary sclerosing cholangitis, 2 had pancreatic carcinoma, 1 had Klatskin tumors, 1 had periampullary carcinoma, 2 had gallbladder carcinoma, 7 patients had cholangiocarcinoma and 1 patient was diagnosed to have obstruction because of metastases. Overall, MRCP was $80 \%$ sensitive, $97.5 \%$ specific and $95.56 \%$ accuracy of in detecting the cause of obstruction.

Conclusion: MRCP is a noninvasive and well tolerated imaging technique in the diagnosis of obstructive jaundice

Keywords: Obstructive jaundice, MRCP, ERCP

\section{INTRODUCTION}

Jaundice is a common problem in both medical and surgical practice. The management of obstructive jaundice poses diagnostic and therapeutic challenges to surgeons practicing in resource limited countries. Clinical data such as history, physical examination, and laboratory tests can identify patients with obstructive jaundice. However, complete assessment of extrahepatic obstruction often requires the use of various imaging modalities to confirm the biliary obstruction by detecting biliary dilatation, its exact location, probable cause and the extent of disease to aid in treatment planning.

Anumber ofimaging modalities are available for the evaluation of obstructive jaundice. Current technologies include trans abdominal ultrasound (US), endoscopic retrograde cholangiopancreatography (ERCP), endoscopic ultrasound (EUS), percutaneous transhepatic cholangiopancreatography (PTC), magnetic resonance cholangiopancreatography (MRCP), multi detector computed tomography (MDCT), and CT cholangiography (CTC). ${ }^{1}$

MRCP is an application of MR imaging that can provide both high quality cross - sectional images of ductal structures and projectional (coronal) images of the biliary tree and pancreatic duct. Unlike ERCP, MRCP is noninvasive and the images are obtained without administration of oral or intravenous contrast agents. ${ }^{2}$ The purpose of this study was to determine the effectiveness of MRCP in the evaluation of obstructive jaundice.

\section{MATERIAL AND METHODS}

This was a prospective study conducted from December 2018 to May 2019 in the department of Radiodiagnosis at Father muller medical college, Mangalore.

Patient selection: Patients in the age group of 21-85 years suspected of obstructive jaundice on the basis of clinical signs, laboratory workup were prospectively included.

\section{Inclusion criteria}

a. Patients clinically suspected of obstructive jaundice.

\section{Exclusion criteria:}

a. All non obstructive cases of jaundice.

b. Patients with obstructive jaundice but having contraindications for MRI.

c. Patient less than 12 years of age.

Ethical clearance for the study was obtained by the institution research and ethical committee. The study included a total of 30 patients of which 21 and 9 were male and female patients respectively. The study age group was between 21 and 85 
years. All cases where pathologically proven cases after surgery. All included cases where evaluated using MRCP. The radiologist was not blinded to the results of other imaging modalities while interpreting MRCP.

Based on the figures available on the sensitivity of MRI in the literature and with $95 \%$ confidence and $20 \%$ precision, the estimated sample size was 30 patients who underwent MRI. The formula used for calculation is $n=Z \alpha^{2} p(1-p) / e^{2}$ Where $\mathrm{n}=$ sample size

$\mathrm{Z} \alpha=1.96$ at $95 \%$ confidence interval and $\mathrm{e}=$ allowable error

Patient Preparation: Fasting was requested for at least 6 hours or pineapple juice was given before the examination to promote gall bladder filling, gastric emptying, and to reduce unwanted fluid signal from the intestine. Patients were instructed to control their breath according to the technician's instructions.

Technique: All MRCP have been acquired on 1.5T superconducting MRI unit (PHILIPS ACHIEVA 16 channel system) using eight channel body coil. Following sequence was used in the study: T1 weighted FLASH (fast low angle shot), T2 weighted FISP (short TR and TE with large Flip Angle), 3D coronal, single shot turbo Spin Echo Sequence (SSFSE) and HASTE.

Image interpretation: The MRI images were transferred to picture archiving and communication system workstation (FMMC, Mangalore). The salient clinically relevant features with suspicion of obstructive jaundice and involvement of primary confluence were used for the statistical analysis in this secondary confluence, assessment of biliary, vascular and nodal involvement and overall resectability was correlated with surgical findings and/or cholangiography. The cases were followed up to surgery and the histopahological diagnoses from the pathology department were sort. The MRCP findings were than compared with the surgical and pathological diagnosis.

\section{STATISTICAL ANALYSIS}

The data were summarized using descriptive statistics like number (No.) and percent (\%). All the variants were analysed and Chi square test/ Fisher test was used as a test of significance. Sensitivity, specificity, positive predictive value, negative predictive value and diagnostic accuracy of MRCP was also analysed. Statistical significance was set at $\mathrm{p}<0.05$ with $95 \%$ confidence intervals. All the statistical calculations were performed using the software, Statistical Package for Social Sciences (SPSS) for windows version16.0.

\section{RESULTS}

All 30 patients underwent MRCP and the final diagnostic tests was done by ERCP in 21 patient and by cytology and histopathological confirmation in 5 patient. Of the 30 patients, 1 patient had a past history of cholecystectomy and 1 pt was known case of carcinoma oesophagus.

In the present study MRCP accurately diagnosed Intra hepatic, CBD dilation in $96.6 \%$ and

93.3\%. 96.7\% accurately diagnosed cases having dilation both intrahepatic as well as

CBD (table-1). In the present case common site of obstruction

\begin{tabular}{|l|c|c|}
\hline Detection of ductal dilatation & MRCP & $\begin{array}{c}\text { Final } \\
\text { diagnosis }\end{array}$ \\
\hline Intrahepatic biliary dilatation & 20 & 19 \\
\hline CBD Dilatation & 4 & 6 \\
\hline $\begin{array}{l}\text { Both CBD \&amp; intrahepatic biliary } \\
\text { dilatation }\end{array}$ & 6 & 5 \\
\hline Total & 30 & 30 \\
\hline $\begin{array}{l}\text { Table-1: Comparison of ductal dilatation by MRCP and con- } \\
\text { firmed by final diagnostic tests (ERCP and / biopsy) }\end{array}$ \\
\hline
\end{tabular}

\begin{tabular}{|l|c|c|}
\hline & $\begin{array}{c}\text { No. of cases } \\
\text { (MRCP) }\end{array}$ & $\begin{array}{c}\text { No. of cases } \\
\text { (Final diagnostic } \\
\text { test) }\end{array}$ \\
\hline Benign lesions & 12 & 10 \\
\hline Stone in CBD and gall bladder & 3 & 5 \\
\hline Benign stricture & 1 & 0 \\
\hline Primary sclerosing cholangitis & 2 & \\
\hline Malignant lesions & 2 & 1 \\
\hline Gall bladder carcinoma & 1 & 2 \\
\hline Pancreatic carcinoma & 7 & 5 \\
\hline Periampullary carcinoma & 1 & 2 \\
\hline Cholangiocarcinoma & 1 & 1 \\
\hline Klatskin tumor & 30 & 30 \\
\hline Metastases & 2 & 1 \\
\hline Total & \multicolumn{2}{|c|}{} \\
\hline Table-2: Pathological diagnosis of the cases by MRCP and by \\
\hline \multicolumn{2}{|c|}{ final diagnostic tests } \\
\hline
\end{tabular}

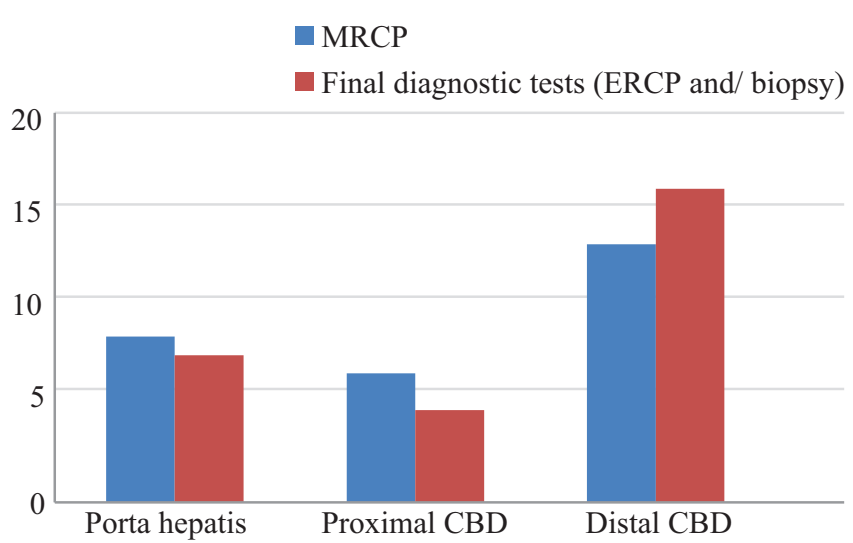

Figure-1: Detection of level of obstruction by MRCP compared to final diagnostic tests (ERCP and /Biopsy)

was at the distal level of CBD (figure-1).

2 cases were falsely diagnosed as choledocholithiasis on MRCP, Biopsy confirmed it to be benign stricture and other to be carcinoma gall bladder. MRCP was unable to diagnose a specific cause for 2 cases where ERCP confirmed the diagnosis to be benign stricture. 1 case of stricture was wrongly diagnosed by the MRCP as cholangitis. In present study 14 patients were diagnosed with malignant lesions by MRCP whereas on ERCP and by biopsy it was 15 cases. MRCP picked up 2 cases of carcinoma pancreas out of which 1 turned out to be periampullary carcinoma. MRCP accurately diagnosed four out of two cases of carcinoma gall bladder. MRCP diagnosed 7 cases as cholangiocarcinoma, out of which 2 cases were later diagnosed by ERCP as Klatskin tumor and other as benign stricture. 
The overall sensitivity, specificity and diagnostic accuracy of MRCP in identifying the cause of obstruction was $80 \%$, $97.5 \%$ and $95.56 \%$ respectively (table- 2 ).

\section{DISCUSSION}

The initial evaluation of obstructive jaundice involves distinguishing intrahepatic and extrahepatic biliary obstruction. Clinical data such as history, physical examination and laboratory tests has been shown to accurately identify upto $90 \%$ of patients whose jaundice is caused by extrahepatic obstruction. ${ }^{5}$ Appropriate management depends upon the identification of patients who would benefit from surgery or a therapeutic intervention. The goal of any radiologic procedure in obstructive jaundice is to confirm the presence of biliary obstruction by detecting biliary dilatation, its exact location, extent and probable cause.

$\mathrm{MRCP}$ is a non -invasive technique used for visualization of the biliary and pancreatic ductal system. MRCP becomes the standard technique of non-invasive investigation of the biliary tree to visualize ductal dilatation, strictures as well as intraluminal filling defects. ${ }^{3}$ Several papers have demonstrated the extremely high diagnostic accuracy of MRCP in the detection of biliary duct stones, benign biliary strictures as well as malignant lesions of the biliary tree, particularly the Klatskin tumor. ${ }^{4-7}$ Ductal dilatation was depicted accurately by MRCP with sensitivity of 95\%, according to a Fulcher AS et al. ${ }^{8}$ Choledocolithiasis is the most common biliary pathology. T2-weighted cholangiography is known to be highly sensitive and specific for detection of biliary filling defects, and for stones in particular. ${ }^{9}$ Postcholecystectomy disorders are rare but are becoming increasingly frequent as a result of the rapid acceptance of laparoscopic cholecystectomy. MRCP is also used to demonstrate postsurgical complications. ${ }^{10}$ A study conducted by Hurter D et al ${ }^{11}$ showed MRCP had sensitivity, specificity, and positive and negative predictive values of $87 \%, 80 \%, 83.3 \%$ and $84.2 \%$, respectively, for bile duct calculi. In this study the sensitivity, specificity and accuracy of $100 \%, 90 \%$ and $93.3 \%$ respectively. MRCP diagnostic accuracy, sensitivity and specificity are comparable to those reported in the literature Calvo et $\mathrm{al}^{12}$, Huassein et al. ${ }^{13}$, and Varghese et al. ${ }^{14}$, where sensitivity, specificity and diagnostic accuracy respectively range between $81-100 \%, 84-100 \%$ and $90-96 \%$. It is known that the sensitivity of MRCP for detecting choledocholithiasis decreases with bile duct dilatation. ${ }^{15}$

In the present case MRCP sensitivity in detecting benign stricture in the present study was $60 \%$ and specificity was $100 \%$. The only case misdiagnosed by MRCP was cholangiocarcinoma confirmed on ERCP. One case of common bile calculus, and another

of cholangitis was confirmed by ERCP, interpreted by MRCP as stricture. Study conducted by Al-Obaidi et al. ${ }^{16}$ showed higher sensitivity (100\%), specificity $(98.5 \%)$, accuracy $(98.7 \%)$ of MRI/MRCP for cases with benign stricture as compared to sensitivity of USG (44.4\%). Verma et al. ${ }^{17}$ demonstrated the sensitivity and specificity of $92.3 \%$ and $86 \%$ on MRCP for detecting the benign etiology of obstruction. Ferrari FS et a $1^{18}$ demonstrated similar findings for benign lesions in their study. The diagnostic accuracy, sensitivity and specificity of MRCP was $93.13 \%, 90 \%, 94 \%$ respectively. MRI is significantly superior to computed tomography in both detecting and excluding malignant conditions. ${ }^{19}$ In the present study MRCP showed 93.3\%, $96.6 \% .96 .6 \%, 93.3 \%, 96.6 \%$ and $100 \%$ accuracy in diagnosing gall bladder carcinoma, pancreatic carcinoma, periampullary carcinoma, cholangiocarcinoma, Klarskin tumor and metastases. The advantages of this technique are that it does not use contrast media or ionizing radiation, it is noninvasive and complication free, allows multiplanar imaging, and the examination is relatively short. Disadvantages of magnetic resonance imaging (MRI) include lack of availability, cost, and difficulties with claustrophobic patients or those with ferromagnetic metallic implants as aneurysm clips. ${ }^{20}$

\section{CONCLUSION}

From this study, we concluded that MRCP is highly accurate in detecting the presence and defining the level of biliary obstruction. MRCP will provide the basis for not only subsequent diagnostic protocol, but also the suitable therapeutic procedure whether surgical or interventional. MRCP have been established in the evaluation of choledocholithiasis, malignant obstruction, congenital anomalies, and postsurgical alterations of the biliary tract.

\section{REFERENCES}

1. Reiner CS, MerkleEM, Bashir MR, Walle NL, Nazeer HK, Gupta RT. MRI Assessment of Biliary Ductal Obstruction: Is There Added Value of T1-Weighted Gadolinium- Ethoxybenzyl-Diethylenetriamine Pentaacetic Acid-Enhanced MR Cholangiography?. AJR: 2013;201(1):49-56.

2. Munir K, Bari V, Yaqoob J, Khan DBA, Usman MU.The Role of Magnetic Resonance Cholangiopancreatography (MRCP) in Obstructive Jaundice. J Pak Med Assoc. March 2004;54(1):128-32.

3. Derrick F. Martin, Hans-Ulrich Laasch. The biliary tree. In: R. G. Grainger, D. J. Allison, A. Adam and A. K. Dixon eds. Grainger and Allison's diagnostic radiology, 4th edition, London, Churchil Livingstone, 2001;2 (3): 1292-1293.

4. Fulcher AS. MRCP and ERCP in the diagnosis of common bile duct stones. Gastrointest Endosc 2002; 56(6 Suppl): S178-S182.

5. Soto JA, Barish MA, Alvarez O, Medina S. Detection of choledocholithiasis with MR cholangiography: comparison of three-dimensional fast spinecho and single- and multisection half-Fourier rapid acquisition with relaxation enhancement sequences. Radiology 2000; 215 (5): 737-745.

6. Demartines N, Eisner L, Schnabel K, Fried R, Zuber M, Harder F. Evaluation of magnetic resonance cholangiography in the management of bile duct stones. Arch Surg 2000; 135 (1): 148-152.

7. Soto JA, Castrillón GA. Clinical applications of magnetic resonance cholangiopancreatography. Radiologia 2007; 49 (3): 389-396.

8. Fulcher AS, Turner, M. A., \&amp; Franklin, K. J., et al. Primary sclerosing cholangitis: evaluation 
with MR cholangiography- a case control study. Radiology.2000;215(1):71-80.

9. Sodickson, A., Mortele, K.J., \&amp; Barish, M. A., et al. Threedimensional fast-recovery fast spin-echo MRCP: comparison with two-dimensional single-shot fast spin echo techniques. Radiology2006; 238(2):549-559.

10. Saisho, H., \&amp; Yamaguchi, T. Diagnostic imaging for pancreatic cancer: computed tomography, magnetic resonance imaging, and positron emission tomography. Pancreas 2004;28(3):273-278.

11. Hurter D, Potgieter PH, Joubert. Accuracy of MRCP compared with ERCP in the diagnosis of bile duct disorders. SA Journal of Radiology 2008;12(1):14-22.

12. Calvo MM, Bujanda L, Calderon A, et al. Role of magnetic resonance cholangiopancreatography in patients with suspected choledocholithiasis. Mayo Clin Proc. 2002; 77 (6): 422-28.

13. Hussein FM, Alsumait B, Aman S, et al. Diagnosis of choledocholithiasis and bile duct stenosis by magnetic resonance cholangiogram. Australas Radiol. 2002; 46 (3): 41-46.

14. Varghese Liddell, et al. MRCP versus US in the detection of choledocholithiasis. Clinical radiology. 2000;55 (5):25-35.

15. Moon JH, Young Deok Cho, Sang Woo Cha, et al. The detection of bile duct stones in suspected biliary pancreatitis: Comparison of MRCP, ERCP, and intraductal US. Am J Gastroenterol 2005;100(4): 10511057.

16. Al-Obaidi S, Al-Hilli MR, Fadhel AA. The Role of Ultrasound and Magnetic Resonance Imaging in the Diagnosis of Obstructive Jaundice. The Iraqi Postgraduate Medical Journal. 2007;6(1):7-17.

17. Verma SR, Sahai SB, Gupta PK, Munshi A, Verma SC, Goyal P. Obstructive Jaundice- Aetiological Spectrum, Clinical, Biochemical And Radiological Evaluation At A Tertiary Care Teaching Hospital. The Internet Journal of Tropical Medicine. 2011;7 (1):12-19.

18. Francesco SF, Federica Fantozzi, Laura Tasciotti, Francesco Vigni, Francesca Scotto, Paolo Frasci. A comparative study in 131 patients with suspected biliary obstruction. Med Sci Monit. 2005; 11(3): 8-18

19. Kim MJ., Mitchell, D. C., \&amp; Ito, K., et al. Biliary dilatation: differentiation of benign from malignant causes- value of adding conventional MR imaging to MR cholangiopancreatography. Radiology 2000;214(1):173-181.

20. Keogan, M. T., \&amp; Edelman, R. R. (2001). Technologic advances in abdominal MR imaging. Radiology 2001; 220(2):310-320.

Source of Support: Nil; Conflict of Interest: None

Submitted: 10-07-2019; Accepted: 23-08-2019; Published online: 20-09-2019 